\title{
Translational control 1995-2015: unveiling molecular underpinnings and roles in human biology
}

\author{
ALAN G. HINNEBUSCH \\ Laboratory of Gene Regulation and Development, National Institute of Child Health and Human Development, National Institutes of Health, \\ Bethesda, Maryland 20892, USA
}

The last 20 years have seen tremendous progress in deciphering the fundamental mechanisms of protein synthesis and molecular pathways of translational control, and in implicating these processes in development, neurobiology, and etiology of human diseases. Much of this success can be attributed to the introduction of new technologies, such as next-generation RNA sequencing and advances in cryo-EM; and also to development of powerful in vitro systems recapitulating complex in vivo phenomena with purified components. Also critical have been strategic interactions between experts in different disciplines of biology, such as the wedding of biochemistry with genetics and structural biology, and of translation biochemistry with neurobiology and medicine. RNA has contributed to this progress by publishing key findings on molecular mechanisms of protein synthesis and the role of translational control in regulating gene expression in diverse organisms.

The basic reactions of the elongation and termination stages of protein synthesis are highly conserved throughout all three kingdoms of life. Because they occur in the catalytic center of the ribosome, their molecular mechanisms were illuminated by the stunning successes of X-ray crystallography and cryo-electron microscopy in producing high-resolution structures of both empty ribosomes and a myriad of ribosome complexes with mRNA, tRNAs, or elongation, termination, or ribosome recycling factors trapped in different stages of the process. Recent innovations in cryo-EM technology are revealing ribosome structures at nearly $3 \AA$ resolution; and new algorithms for sorting structurally distinct molecules means that sample heterogeneity (an anathema to crystallography) is now exploited to identify different conformational states in the same preparation.

The solved ribosome structures revealed the path of mRNA and the A, P, and E binding sites for the tRNA, as it progress through the elongation cycle from aminoacyltRNA, to peptidyl-tRNA to deacylated tRNA, located at the interface between the large and small subunits; as well as

Corresponding author: ahinnebusch@nih.gov

Article and publication date are at http://www.rnajournal.org/cgi/doi/ 10.1261/rna.049957.115. Freely available online through the RNA Open Access option. the peptidyl transferase center where peptide bond formation occurs. As the decoding center of the ribosome is comprised almost entirely of rRNA, it became clear that the ribosome is a ribozyme. The protein factors involved in translocation of mRNA and the tRNAs through the decoding sites (EF-G/ EF2), in recognizing stop codons and hydrolyzing peptidyltRNA (RF1/eRF1), and splitting apart ribosomal subunits following termination (bacterial RRF) all exploit striking structural mimicry of tRNA to fit into the A site. Interestingly, RF1 was shown to contain a protein element that "reads" the stop codon and another that reaches into the peptidyl transferase center to hydrolyze the peptidyl-tRNA linkage. The peptidyl-tRNA and aminoacyl-tRNA are anchored in the precise locations required for peptide bond formation, but the ribosome is not simply a rigid catalytic platform, but undergoes conformational changes in selecting the correct EF1A.GTP-aminoacyl-tRNA ternary complex for each codon, and displays inter-subunit ratcheting during translocation and termination. GTP binding and hydrolysis by the elongation factors evoke cyclic conformational changes that drive the process forward. The use of fluorescently labeled reactants and stopped-flow kinetic analysis allowed a detailed dissection of different steps in the processes of aminoacyltRNA selection in the A site, with proofreading of the codon:anticodon interaction; and of translocation of tRNAs through the decoding center catalyzed by EF2. Single-molecule approaches also contributed importantly to this progress, by identifying intermediate states too transient to be detected by ensemble kinetic experiments or structural biology. In other developments, eIF5A and its bacterial counterpart EF-P were shown to stimulate elongation specifically through stretches of proline codons, and both factors occupy the E-site and communicate directly with the peptidyl transferase center. Interestingly, RRF is replaced in eukaryotes and archaea by an ATP-binding cassette protein, ABCE1/ Rli1, that becomes wedged between the large and small subunits and uses ATP hydrolysis to split apart the subunits. ABCE1/Rli1also functions with RF1 paralog Dom34 to

This article, published in $R N A$, is available under a Creative Commons License (Attribution-NonCommercial 4.0 International), as described at http://creativecommons.org/licenses/by-nc/4.0/. 
dissociate empty $80 \mathrm{~S}$ couples and stalled elongating $80 \mathrm{~S}$ subunits in mRNA surveillance pathways.

Twenty years ago, the broad outlines of eukaryotic initiation by the scanning mechanism were understood, and the major players identified. Since then, crystallography and NMR have provided atomic structures of eIF1, eIF1A, and the heterotrimeric GTPase eIF2 (or its archaeal counterpart) in a ternary complex with GTP and methionyl initiator tRNA $\left(\mathrm{tRNA}_{\mathrm{i}}\right)$; and also defined the locations of these factors on the $40 \mathrm{~S}$ subunit in preinitiation complexes (PICs) with mRNA. A combination of yeast genetics and biochemical analysis in reconstituted yeast and mammalian systems has revealed that eIF1 and eIF1A promote a scanning-conducive conformation of the PIC with $\mathrm{tRNA}_{\mathrm{i}}$ not fully engaged with the $\mathrm{P}$ site but capable of sampling triplets for complementarity to the anticodon. eIF1 was identified as the key "gatekeeper" that restricts initiation to AUGs in good context, and must dissociate from the PIC, for stable tRNA $\mathrm{A}_{\mathrm{i}}$ binding in the $\mathrm{P}$ site. This and other rearrangements involving the tails of eIF1A allow completion of GTP hydrolysis by eIF2 $\left(\mathrm{P}_{\mathrm{i}}\right.$ release $)$ and a halt to further scanning. The eIF5 also promotes these rearrangements in addition to being the GTPase activating protein for eIF2. These advances depended on the use of "toe-print" assays to map PIC locations on the mRNA, and the development of assays for measuring rates of eIF1 and $\mathrm{P}_{\mathrm{i}}$ dissociation, and of changes in relative proximity of eIFs within the PIC using fluorescent probes, in real time. Analysis of mutants showed that the integrity of all of these eIFs is crucial to prevent aberrant initiation at non-AUG codons in cells; and it was learned that the functions of eIF1 and eIF5 are exploited to autoregulate their own translation. We know now that the multi-subunit eIF3 complex embraces the solvent-exposed side of the $40 \mathrm{~S}$ subunit, contacting mRNA where it enters and exits the ribosome, and also reaches into the subunit interface side of the PIC to contact eIFs 1, 2, and 5 and support their functions in scanning and AUG selection. A combined assault by many labs and approaches is starting to crack the 3-D structure of eIF3 and its ribosome interface, but much is still unknown about the molecular basis of eIF3 function.

It was understood in 1994 that PIC attachment to mRNA requires the helicase eIF4A and its binding partner eIF4G, which are tethered to the mRNA $5^{\prime}$ cap by eIF4E. Since then, we've learned that eIF $4 \mathrm{G}$ is multifunctional, promoting the active conformation of eIF4A; forming a stable, circularized mRNP through separate binding domains for RNA and the poly(A) tail binding protein (PABP); and enhancing PIC attachment through interactions with eIF3, eIF5, and eIF1. While once considered to be merely a cofactor for eIF4A, it is now believed that eIF4B stimulates PIC-mRNA association when bound near the $40 \mathrm{~S}$ entry channel and, at least for mammalian eIF4B, increases the efficiency of coupling ATP hydrolysis to mRNA unwinding. Other helicases, Ded1/Ddx3 and Dhx29, were implicated in scanning through stable RNA secondary structures, but it's unclear how much their functions overlap with one another and eIF4A in vivo. Although the PABP.eIF4G interaction is only stimulatory and non-essential, it likely enhances eIF4G competition with non-specific mRNA binding proteins (RBPs), and helps to explain how lengthening or shortening the poly $(\mathrm{A})$ tail by cytoplasmic poly(A) polymerases and ribonucleases regulates translational efficiency in oocytes. Finally, it was discovered that the eukaryotic homolog of bacterial IF2 is recruited to the PIC by eIF1A where it catalyzes joining of the 60S subunit to the PIC following AUG recognition, completing assembly of the $80 \mathrm{~S}$ initiation complex. There is enormous potential for applying single-molecule approaches to studying the mechanics of ribosomal scanning and identifying transient intermediates along the initiation pathway.

Internal ribosome entry sites (IRESs) in picornaviruses were discovered in the late 1980s, but the last two decades saw the reconstitution of viral IRES function in purified systems, as well as structural elucidation of ribosome-IRES complexes. We now know that viral IRESs use complicated RNA structures, in some cases stabilized by accessory RNAbinding proteins (ITAFs), to interact with, and manipulate, the $40 \mathrm{~S}$ subunit and thereby dispense with canonical eIFs to differing extents. This trend culminates in IRESs that require no eIFs or $\mathrm{tRNA}_{\mathrm{i}}$ at all and use a pseudoknot to mimic tRNA-mRNA interaction-making them resistant to host defenses that attenuate canonical eIF function to limit virus propagation. A long list of IRESs in host cell mRNAs has emerged, although none has been functionally reconstituted in vitro, and some may be artifacts of the dicistronic reporters used to detect them. There are indications that overexpression of eIF4G or inhibition of eIF4E, favoring cap-independent initiation, stimulates some cellular IRESs. Other intriguing unconventional initiation pathways were uncovered including initiation at CUG codons by a leucyltRNA — which might underlie production of peptides for immune surveillance-and initiation at non-cognate codons in trinucleotide repeat tracts associated with certain human disease mutations.

Recruitment of $40 \mathrm{~S}$ subunits by certain IRESs is functionally equivalent to the role of the short Shine-Dalgarno (SD) sequence in bacterial mRNAs, located $\sim 8 \mathrm{nt}$ upstream of the AUG, which base pairs to 16S rRNA to recruit the $30 \mathrm{~S}$ subunit to the initiation codon. It was shown that another eukaryotic SD-analog is the TURBS element that promotes reinitiation in bicistronic caliciviruses mRNAs by base pairing to $18 \mathrm{~S}$ rRNA to allow $40 \mathrm{~S}$ subunits to remain attached and reinitiate at the nearby AUG of the second cistron. The low efficiency of reinitiation after translating a long ORF (in the absence of something like TURBS) likely involves the absence of eIF3 on post-termination 40S subunits, which can be mitigated for short uORFs by eIF4G.eIF3 interaction or, as for uORF1 in GCN4 mRNA, eIF3-mRNA contact. It was known that reinitiation is regulated for yeast GCN4 mRNA by reducing availability of the eIF2.GTP.tRNA $A_{i}$ TC via eIF2 $\alpha$ phosphorylation by kinase Gcn2, which enables 
scanning ribosomes that translated uORF1 to bypass inhibitory uORFs 2-4 and reinitiate further downstream at the main ORF. This mechanism has been extended to mammalian ATF4 mRNA, where it mediates the integrated stress response. In other mRNAs, uORFs regulate translation differently, as the uORF peptide product stalls the elongating ribosome by interacting unfavorably with the exit tunnel of the ribosome-in a manner that can be modulated by small molecules.

eIF2 $\alpha$ phosphorylation was the first known mechanism for down-regulating bulk initiation in stress situations, which involves inhibiting eIF2's GEF, eIF2B. Three of 5 eIF2B subunits were shown to bind phosphorylated eIF2 and impede the function of the catalytic moiety at the C-terminus of the $\varepsilon$-subunit. The eIF2 GAP, eIF5, also contains a GDP-dissociation-inhibitory domain that impedes eIF2B access to eIF2-GDP and enhances the inhibitory effect of eIF2 $a$ phosphorylation on GDP-GTP exchange by eIF2B. Gcn2 is activated in amino acid-starved cells by its binding of uncharged tRNA, which likely occurs on elongating ribosomes, is facilitated by Gcn1 and Gcn20, and evokes allosteric activation of the intrinsically inactive kinase domain (KD). Dimerization of the KD is crucial for activation of Gcn2, and for eIF2 $\alpha$ kinases PKR and PERK, being triggered, respectively, by dsRNA in virus-infected cells and unfolded ER proteins. Structural analysis of the PKR·eIF2a complex illuminated the molecular basis for the exquisite substrate specificity of eIF2a kinases.

A second major mechanism of global translational control was discovered involving the $4 \mathrm{E}-\mathrm{BP}$ proteins that bind eIF4E and displace eIF4G by mimicking the eIF4E-binding site of eIF4G. Phosphorylation of mammalian 4E-BPs by mTOR dissociates them from eIF4E and promotes increased translation in response to nutrients and growth factors, and has been implicated in the localized protein synthesis stimulated at neuronal synapses. The same mechanism of steric hindrance of eIF4G-eIF4E assembly is utilized by translational repressors with key roles in animal development, which are targeted to specific mRNAs by $3^{\prime}$ UTR-bindng RBPs. A related mechanism involves tethering at the cap of a "dominant negative" eIF4E-related protein incapable of binding eIF4G. Other translational repressors were uncovered that bind to the $3^{\prime}$ UTR and target PIC attachment to the eIF4E-eIF4G complex, such as Drosophila Sxl (represses MSL-2 mRNA involved in sex determination) and mammalian GAIT complex (mediates interferon repression of certain inflammatory gene mRNAs), or impair subunit joining, as in hnRNPs K/ E1 repression of 15-lipoxogenase mRNA. Pat1, RCK/Dhh1, and RAP55/Scd6 were identified as translational co-repressors that can be recruited to specific mRNAs by other RBPs, or effect global translational repression, in a variety of organisms and circumstances by poorly understood mechanisms; and Pat1 also strongly stimulates mRNA decapping by Dcp2/Dcp1. A major discovery was that microRNAs also target $3^{\prime} \mathrm{UTR}$ and mediate translational repression via
Ago-GW182 protein complexes, which is frequently accompanied by deadenylation and mRNA degradation; however, the molecular mechanism(s) of translational repression still remain to be fully elucidated. This is a critical goal because a huge proportion of the mammalian transcriptome is likely under miRNA control.

The role of mRNA localization in restricting translation to specific subcellular components has continued to grow in importance in developmental pathways of early embryogenesis, and in dendrites and axons (which can be separated by huge distances from the cell body/nucleus) in neuronal wiring and function. It was established that RBPs mediate both transport, via connections to motor proteins, and translational repression en route to the correct cellular location, such as ZBP1 for $\beta$-actin mRNA. Certain RBPs have self-aggregation properties that could underlie assembly of mRNP granules involved in mRNA localization or mRNA storage/turnover during stress, such as P-bodies and stress granules.

It's difficult to understate the importance of the technological advance of deep-sequencing ribosome-protected mRNA fragments (ribosome footprint profiling) to achieve a snapshot of the locations of all translating $80 \mathrm{~S}$ ribosomes, and measure translational efficiencies of mRNAs genome-wide. One can follow how the cellular "translatome" changes in response to external stimuli, alterations in levels/functions of translation factors, or in disease states. Application of the technique has revealed 5'UTR translation, often initiated at near-cognate triplets, at higher levels than anticipated, and also the presence of $80 \mathrm{~S}$ ribosomes in the $3^{\prime} \mathrm{UTR}$ in cells lacking the recognition of idle $80 \mathrm{~S}$ subunits by Dom34. And by its ability to reveal translation reading frame, ribosome profiling has identified translating ribosomes in certain non-coding RNAs, increasing the appreciated protein-coding potential of the genome.

Finally, it has become increasingly clear that a number of human diseases involve mutations in general translation factors that have surprisingly tissue-specific pathologies, frequently in the nervous system, such as eIF2B and eIF2 mutations associated with Vanishing White Matter disease and a form of intellectual disability, and mutations in the enzyme Dyskerin, which modifies $~ 100$ uridines in rRNA, responsible for X-linked dyskeratosis congenita. These nonlethal reductions in initiation factor or ribosome function might alter the translation of specific mRNAs whose products are critically important in the affected tissues. Mutations in RBPs also underlie defects in neural development, such as mutations in FMRP responsible for Fragile $\mathrm{X}$ mental retardation, which impair translational repression (likely during elongation) of a large cohort of mRNAs. Accumulating evidence indicates that altered expression of initiation factors contributes to cancer; eg. overexpression of eIF4E is oncogenic, and tumors frequently exhibit highlevel eIF4E. Translational control via eIF2 $\alpha$ phosphorylation also appears to be important for cancer cells to survive 
hypoxia. An important goal will be to identify the specific mRNAs whose altered translation is responsible for different diseases, a task for which ribosome profiling seems ideally suited.

Many of the advances of the past 20 years have been fueled by technological advances and collaborations among workers with distinct, complementary skills and, thus, would have been difficult to foresee in 1994. I sense that the pace of inno- vation and synergy are accelerating in our field, as in other research disciplines, so that an observer in 2035 will be even more impressed than I have been with the expansive progress of the last two decades. I also feel certain that, in 2035, RNA will still be viewed as one of the best forums for interdisciplinary research on the structure and function of mRNA, the machinery of protein synthesis, and pathways of translational control, in all branches of life. 

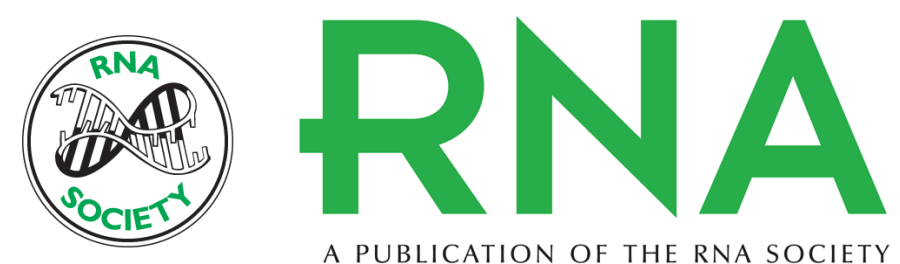

A PUBLICATION OF THE RNA SOCIETY

\section{Translational control 1995-2015: unveiling molecular underpinnings and roles in human biology}

Alan G. Hinnebusch

RNA 2015 21: 636-639

Open Access Freely available online through the RNA Open Access option.

Creative This article, published in RNA, is available under a Creative Commons License

Commons (Attribution-NonCommercial 4.0 International), as described at

License http://creativecommons.org/licenses/by-nc/4.0/.

Email Alerting Receive free email alerts when new articles cite this article - sign up in the box at the Service top right corner of the article or click here. 